\title{
Enfermedad renal oculta en pacientes con rasgo drepanocítico
}

\author{
Brian M. Erazo ${ }^{1}$ \\ Gilberto A. Ramírez, Nestor J. Yanes, Linda E. Cerrato, \\ Isis A. Buezo, Fabiola C. Martel ${ }^{2}$ \\ Gustavo A. Fontecha ${ }^{3}$
}

\section{RESUMEN}

El rasgo drepanocítico es casi siempre visto como una condición benigna pero se ha demostrado que puede causar diferentes consecuencias patológicas. Dentro de esta asociación, una de las más importantes, es el riesgo aumentado de enfermedad renal crónica en estos individuos. El presente estudio tiene como objetivo determinar la prevalencia de enfermedad renal oculta en personas identificados con rasgo drepanocítico. Se realizó un estudio descriptivo y transversal en una muestra de la población de la comunidad de Ciriboya, del departamento de Colón, Honduras en enero del año 2015. Participaron 20 personas, mayores de 18 años, las cuales fueron identificadas con rasgo drepanocítico en una investigación previa. Para calcular el riesgo se utilizó la herramienta validada de tamizaje para Enfermedad Renal Oculta (SCORED), se determinaron los niveles de hemoglobina, glucosa en ayunas, tensión arterial y proteinuria, así mismo se registraron antecedentes patológicos y factores de riesgo de cada persona. Los datos fueron ingresados y procesados en el programa Epi info 7 y el nivel de riesgo para enfermedad renal fue calculado mediante los puntajes que este instrumento establece. La muestra estudiada fue de 13 mujeres (65\%) y 7 hombres (35\%) en un rango de edad entre 19 y 83 años con una SD de 18.4, se encontró una prevalencia de riesgo aumentado de enfermedad renal en el $45 \%$ de los sujetos de estudio. Las personas con rasgo drepanocítico deben de recibir un seguimiento clínico especial considerando el riesgo aumentado de padecimiento de enfermedad renal en la población estudiada

Palabras clave: rasgo drepanocítico, enfermedad renal, factores de riesgo.

\footnotetext{
${ }^{1}$ Estudiantes egresados de la Carrera de Medicina, Facultad de Ciencias Médicas, UNAH: brian.erazo@unah.hn.

${ }^{2}$ Estudiantes egresadas de la Carrera de Microbiología, Facultad de Ciencias, UNAH.

${ }^{3}$ Asesor. Profesor del Instituto de Investigaciones en Microbiología, Facultad de Ciencias, UNAH: gustavo.fontecha@unah.edu.hn.
} 


\section{ABSTRACT}

Sickle cell trait is usually seen as a benign condition, but has been shown to cause different pathological consequences. Within these associations, one of the most important is the increased risk of chronic kidney disease in these individuals. This study aims to determine the prevalence of occult renal disease identified in people with the condition. The study was conducted in the population of the community of Ciriboya, Colón, Honduras in January 2015. With a sample of 20 people, over 18 years, which were identified with sickle cell trait in a previous research. Validated screening tool for Occult Renal Disease (SCORED) was applied in each participant, hemoglobin levels, glucose, blood pressure and proteinuria were determined, also medical history and risk factors were recorded. Data were entered and processed in the software Epi info 7. The sample included 13 women (65\%) and 7 men (35\%) in age ranges between 19 and 83 years with a SD of 18.4, in which the prevalence of increased risk for kidney disease was found in $45 \%$ of the study subjects. To give a special attention to people with sickle cell trait, since it is no longer considered a harmless condition, under the circumstances of the increase in pathological complications it can cause. Tracking those identified with increased risk of kidney disease is recommended.

Keywords: Sickle Cell Trait, Kidney Diseases, Risk Factor. 


\section{INTRODUCCIÓN}

Las hemoglobinopatías son un problema en ascenso y con alta prevalencia en los países tropicales y/o en vías de desarrollo. Aproximadamente un $5 \%$ de la población mundial es portadora de genes causantes de hemoglobinopatías, entre las que destacan la drepanocitosis y la talasemia. Estas cifras oscilan desde 5 a 25\% según la región estudiada. Se calcula que cada año nacen en todo el mundo más de 300 mil niños con formas graves de estas enfermedades, la mayoría de ellos en países de bajos y medianos ingresos. La drepanocitosis es la más prevalente de estas patologías, siendo las poblaciones afrodescendientes especialmente vulnerables a este padecimiento (Williams y Weatherall, 2012).

La drepanocitosis 0 anemia de células falciformes es una enfermedad que afecta a millones alrededor del mundo, es un desorden causado por una mutación monogénica en el gen de la $\beta$ globina que produce Hemoglobina S (Pace, 2012). Cuando la mutación está presente de manera heterocigota, en uno de los alelos, la condición se denomina rasgo drepanocítico (HbAS), esta afecta aproximadamente a 300 millones de personas en todo el mundo (Key y Derebail, 2010, p.418).

En Honduras no había estudios sobre este tema, hasta que en el año 2014 se realizó una investigación en Ciriboya, una comunidad de la etnia garífuna ubicada en la costa atlántica de Honduras, con el objetivo de buscar la prevalencia de la mutación genética asociada con drepanocitosis. Los resultados de este estudio reportaron cifras altas del genotipo portador $\beta A / S$ (rasgo drepanocítico), las que ascendían al $23.6 \%$ de la población (Erazo et al., 2015).

La alta prevalencia de rasgo drepanocítico encontrada en esta población hace necesaria la realización de estudios adicionales ya que esta condición no es considerada tan benigna como en el pasado se planteaba. Se ha demostrado que puede causar diferentes consecuencias patológicas (Tsaras, et al., 2009; Ajayi, 2005). Dentro de estas asociaciones patológicas, una de la más importantes es el riesgo aumentado de enfermedad renal crónica (Naik et al., 2014). Por lo que resulta pertinente y urgente realizar un proceso de monitoreo en poblaciones donde hay una alta prevalencia de la condición drepanocítica, desde una visión de prevención, para disminuir el riesgo de desarrollar este padecimiento crónico o proporcionar un tratamiento oportuno a aquellos que ya poseen la enfermedad. Esto se puede hacer mediante instrumentos de tamizaje bastante sencillos, como el tamizaje para enfermedad renal oculta (SCORED), que ha mostrado ser una herramienta con alto valor predictivo y 
costo-efectiva (Bang et al., 2007). El presente tiene como objetivo determinar el riesgo de desarrollar enfermedad renal oculta en personas identificadas con rasgo drepanocítico. Esto contribuirá a dar un seguimiento basado en evidencia de los portadores de esta condición en los establecimientos de salud correspondientes para prevenir el desarrollo de complicaciones.

\section{METODOLOGÍA}

Área de estudio: Se realizó un estudio descriptivo en una muestra de la población de la comunidad de Ciriboya, municipio de Iriona, departamento de Colón, Honduras durante el mes de enero del año 2015.

Unidad de análisis: Los sujetos de estudio fueron 20 personas, mayores de 18 años, previamente diagnosticadas con rasgo drepanocítico mediante la técnica de PCR-RFLP. Estos fueron seleccionados a partir de un estudio previo de esta condición, el cual tuvo un muestro tipo probabilístico aleatorio simple (Erazo et al., 2015). Recolección de datos: La recolección de los datos se realizó mediante la herramienta validada de tamizaje para Enfermedad Renal Oculta (SCORED) (Bang et al., 2007) (Figura1). Siguiendo los criterios de la misma, se midieron los siguientes parámetros: Parámetros de entrevista: Nombre, edad, sexo y si tenía alguno o varios de los siguientes padecimientos diagnosticados previamente por un médico certificado: vasculopatía hipertensiva, antecedente de infarto agudo al miocardio o enfermedad cerebrovascular, insuficiencia cardiaca y vasculopatía extremidades inferiores. Se entrevistó a cada persona de manera individual, estructurada, oral y en privado. Se procuró que cada participante estuviera en un ambiente cómodo, donde este pudiera escuchar, entender y responder con claridad. Así mismo se obtuvo el consentimiento informado y explicó las instrucciones de la participación un día previo a la recolección de datos.

Posteriormente se procedió a la medición de los siguientes parámetros laboratoriales: Hemoglobina y hematocrito. Para la determinación de la hemoglobina y el hematocrito, alrededor de $50 \mathrm{uL}$ de sangre se transfirieron desde los recipientes EDTA a capilares desechables de vidrio de $75 \mathrm{~mm}$ de largo y $1.2 \mathrm{~mm}$ de diámetro, lo suficiente para cubrir tres cuartas partes de su largo total y luego fueron sellados utilizando un compuesto especial para este fin. Seguidamente los capilares fueron colocados en una centrifugadora y girados a 10,000 rpm durante cinco minutos. Se procedió a la lectura utilizando un lector de triangulación comercial de hemoglobina. Luego de la 
cuantificación de la hemoglobina, la interpretación de la presencia de anemia y su magnitud fue hecha utilizando las guías de la Organización Mundial de la Salud (OMS) para este propósito (WHO, 2000 p.1-9) (OMS, 2011, p.1-19). Glicemia. La determinación de este parámetro se realizó mediante muestra de sangre capilar. Se le pidió un día previo a la toma de la muestra a los participantes que estuvieran en ayunas para que el parámetro fuera válido. Se extrajo alrededor de 100 ul de sangre capilar de la falange distal del dedo índice de la mano izquierda, luego se depositó en una tira reactiva, la lectura posteriormente se realizó mediante un glucómetro. Si ocurría error en la prueba, esta se repetía. La presencia de hiperglicemia se indicó si el valor de glucosa en sangre era igual o mayor a $126 \mathrm{mg} / \mathrm{dl}$.

Proteinuria. La medición de este parámetro se realizó en orina, con el paciente en ayunas, con instrucciones previas de higiene dadas a cada participante para la recolección de la muestra. Cada uno depósito de 30 a $80 \mathrm{ml}$ de orina en un frasco estéril. Las muestras fueron transportadas y procesadas en el laboratorio de microbiología del hospital Garífuna. La detección se realizó mediante tiras reactivas de orina y su lectura mediante una interpretación cualitativa con tres observadores distintos. Análisis de datos: Toda la información obtenida se almacenó y procesó en el software Epi info 7. A cada una de las variables de la escala se le asignó un valor de 0 a 4 según los parámetros estandarizados del instrumento. Se interpretó que la existencia de alta probabilidad de enfermedad renal oculta si la sumatoria total de estos parámetros era 4 o mayor. (Ver figura 1)

Consideraciones éticas: El estudio fue aceptado por el hospital Garífuna de Honduras y el Departamento de Ciencia y Tecnología de la Secretaría de Salud. Cada individuo accedió a participar previo a la lectura, discusión y firma de un documento de consentimiento informado. Se cumplieron todos los parámetros de bioseguridad para proteger en cada etapa del proceso a los participantes.

\section{RESULTADOS}

Veinte individuos afrodescendientes diagnosticados previamente como portadores de rasgo drepanocítico, residentes de Ciriboya, municipio de Iriona, departamento de Colon, Honduras, participaron en el estudio. Teniendo un promedio de edad de 55.5 años con una desviación estándar de $18.4 \pm$ (rango entre 19 y 83 años). Ambos sexos representados como sigue: $65 \%$ mujeres y $35 \%$ hombres. 
Figura 1. SCORED (Tamizaje para enfermedad Renal Oculta)

\begin{tabular}{|l|l|}
\hline Parámetro & Puntaje \\
\hline Edad: & 2 \\
1. Entre 50 y 59 años & 3 \\
2. Entre 60 y 69 años & 4 \\
3. 70 años o más & 1 \\
Mujer & 1 \\
Anemia & 1 \\
Hipertensión arterial & 1 \\
Diabetes & 1 \\
Historia de infarto al miocardio o accidente cerebrovascular & 1 \\
Historia de insuficiencia cardiaca & 1 \\
Vasculopatía de miembros inferiores & 1 \\
Proteinuria & $0-12$ \\
Total & \\
\hline Interpretación & \\
4 o más puntos & \\
20 por ciento de probabilidad de enfermedad renal presente. Se recomienda \\
evaluación de estudios adicionales. \\
De 0 a 3 puntos \\
Escasa probabilidad de tener enfermedad renal, se recomienda evaluación \\
anual.
\end{tabular}

Fuente. Traducción al español: Elaboración propia del autor

En la siguiente tabla se describen los hallazgos de los parámetros de tamizaje encontrados:

Tabla 1. Distribución de parámetros de herramienta SCORED en pacientes con rasgo drepanocítico de la comunidad de Ciriboya, Honduras

\begin{tabular}{|l|c|c|}
\hline \multicolumn{1}{|c|}{ Parámetro } & Frecuencia (n) & Porcentaje (\%) \\
\hline Mujer & $(13 / 20)$ & 65.0 \\
Anemia & $(9 / 20)$ & 45.0 \\
Hipertensión arterial & $(8 / 20)$ & 40.0 \\
Diabetes & $(5 / 20)$ & 25.0 \\
Historia de infarto al miocardio 0 accidente cerebrovascular & $(2 / 20)$ & 10.0 \\
Historia de insuficiencia cardiaca & $(3 / 20)$ & 15.0 \\
Vasculopatía de miembros inferiores & $(3 / 20)$ & 15.0 \\
Proteinuria & $(6 / 20)$ & 30.0 \\
\hline
\end{tabular}

